\title{
CHIRONOMID TYPES AT MUSÉUM NATIONAL D'HISTOIRE NATURELLE, PARIS (Meigen collection not included)
}

\author{
By Torbjørn Ekrem \\ Museum of Zoology, University of Bergen, Muséplass 3, N-5007 Bergen, Norway \\ (email: Torbjorn.Ekremoo.uib.no)
}

The chironomid type collection in Paris is, except for the Meigen collection, not very well known. The list presented here was generated during my visit to the Muséum National d'Histoire Naturelle as part of the European Union's PARASYST program in June/July 2000. Notes on the current taxonomic status of the species are included. These notes are, when not available on the labels, collected from the catalogues of Neotropical, Palaearctic, Oriental and Afrotropical Chironomidae (SUBLETTE \& Sublette 1973; FREEMAN \& CRANSTON 1980; ASHE \& CRANSTON 1990; SPIES \& REISS 1996). A list of the chironomid specimens in the Meigen collection, which is currently under revision by Martin Spies (pers. comm.), has previously been published by FITTKAU and REISS (1976).

The specimens of Goetghebuer and Kieffer all bear identical "cotype" labels. These are probably not original, and have been placed on their respective pins by another scientist. Freeman and Macquart types are located in separate boxes. The other type specimens form part of the general chironomid collection.

\section{Acknowledgements}

I would like to thank Dr. Cristophe Daugeron at Muséum National d'Histoire Naturelle for his hospitality during my stay and Prof. Daniel F. Goujet and Dr. François Bouvier for answering my many questions and organizing my visit. Thanks also to Martin Spies for all helpful information and to Trond Andersen for comments on the manuscript.

\section{References:}

Ashe, P. \& Cranston, P.S. (1990). Family Chironomidae. pp 113-355 in SoÓs, A. \& PAPP, L. (eds): Catalogue of Palaearctic Diptera. Volume 2. Psychodidae Chironomidae. Budapest, Akadémiai Kiadó.

Blanchard, E. (1852). Orden IX. Dípteros. pp 1471 in Gay, C. (ed): Historía física y política de Chile. Zoología. Paris \& Santiago de Chile.

FitTKAU, E.J. \& REISS, F. (1976). Die Chironomidentypen und ihr Erhaltungszustand in der Sammlung des Muséum d'Histoire Naturelle, Paris. Chironomus, 1: 146-150.

FREEMAN, P. (1956). Some Chironomidae (Diptera) from French West- Africa. Bull. Inst. fr. Afr. noire, 18: 93-96.

FREEMAN, P. (1961). A collection of Chironomidae and Culicidae subfamily Dixinae (Diptera, Nematocera) from Madagascar. Mém. Inst. scient. Madagascar, Sér. E, 12: 238-255.

Freeman, P. \& Cranston, P.S. (1980). II. Family Chironomidae. Pp 175-202 in CROSSKEY, R. W. (ed): Catalogue of the Diptera of the Afrotropical Region. London, British Museum (Natural History).

Goetghebuer, M. (1919). Observations sur les larves et les nymphes de quelques Chironomides de Belgique. Annls Biol. lacustre, 9: 51-78.

Kieffer, J.J. (1912). H. Sauter's Formosa Ausbeute. Suppl. Ent., 1: 32-43. 
KIEFFER, J.J. (1922). Chironomides nouveaux ou peu connus de la région paléarctique. Annls Soc. scient. Brux., 42: 71-180.

MACQUART, J. (1826). Insectes Diptères du Nord de la France. I. Tipulaires. Mém. Soc. Sci. Agric. Lille, 1823-1824: 59-224.

MACQUART, J. (1838). Diptères exotiques nouveaux ou peu connus. Mém. Soc.Sci. Agric. Lille, 2: 9-225.

R o s s A R O, B. \& Delettre, Y.R. (1992). Description of Smittia celtica, n. sp. (Diptera, Chironomidae). Annls Soc. ent. Fr., 28: 365-370.

SÉGUY, E. (1965). Deux noveaux Tendipédides des Iles Crozet (Insectes Diptères Nématocères. Bull. Mus. natn. Hist. nat., Paris, 37: 285289.
SERra-Tosio, B. (1982). Description du male de Belgica albipes (SÉGUY, 1965), n. comb., rare chironomidé microptère des iles Crozet (Diptera).Revue fr. Ent., 4: 97-100.

SERRA- TOSIO, B. (1986). Un nouveau chironomide antarctique des îles Crozet, Parochlus crozetensis n. sp. (Diptera, Nematocera). Nouv. Revue Ent. (N.S.), 3: 149-159.

SPIES, M. \& REISS, F. (1996). Catalog and bibliography of Neotropical and Mexican Chironomidae (Insecta, Diptera). Spixiana Suppl., 22: 61-119.

Sublette, J.E. \& Sublette, M.F. (1973). Family Chironomidae. Pp 389-422 in Delfinado, M. D. \& Hardy, D. E. (eds): A catalog of the Diptera of the Oriental region. Honolulu, The University Press of Hawaii.

\section{Table 1. Types in the general chironomid collection in Muséum National d'Histoire Naturelle, Paris}

\begin{tabular}{|c|c|c|c|c|c|}
\hline Number & Species & Author & Type status & Synonyms/present genus & Locality \\
\hline 21 & "nitidus" & "MACQ" * & "Type", good* & $\begin{array}{l}\text { "Paratendipes albimanus, } \\
\text { GTGH." }\end{array}$ & Not given \\
\hline \multirow[t]{9}{*}{3683} & Belgica albipes & (SÉGUY, 1965) & $\begin{array}{l}\text { Allotype male } \\
\text { det. Serra-Tosio } \\
\text { (1982) on slide }\end{array}$ & & $\begin{array}{l}\text { Crozet: Baie du } \\
\text { Navirre Rochers, } \\
\text { 25.III.1972, Ph. Dreux }\end{array}$ \\
\hline & Chaetocladius angustus & FREEMAN, 1961 & $\begin{array}{l}\text { Holotype, male, } \\
\text { good }\end{array}$ & $\begin{array}{l}\text { Bryophaenocladius } \\
\text { angustus FrEEMAN, } 1961\end{array}$ & $\begin{array}{l}\text { Madagascar Centre, } \\
\text { Pic Boby } 2400 \mathrm{~m}, \\
\text { Andringitra, } \\
\text { Ambalavao, 11- } \\
\text { 14.I.1958, B. } \\
\text { Stuckenberg }\end{array}$ \\
\hline & Chaetocladius angustus & FREEMAN, 1961 & $\begin{array}{l}\text { Paratype, male, } \\
\text { good }\end{array}$ & & As holotype \\
\hline & Chironomus bequaerti & $\begin{array}{l}\text { GOETGHEBUER, } \\
1921\end{array}$ & $\begin{array}{l}\text { Cotype, male, } \\
\text { good }\end{array}$ & $\begin{array}{l}\text { Einfeldia longipes } \\
\text { STAEGER, } 1839\end{array}$ & $\begin{array}{l}\text { Destelbergen, } \\
\text { 11.V.1915, M. } \\
\text { Goetghebuer }\end{array}$ \\
\hline & Chironomus brayi & $\begin{array}{l}\text { GOETGHEBUER, } \\
1921\end{array}$ & $\begin{array}{l}\text { Cotype, female, } \\
\text { good }\end{array}$ & Nilothauma brayi & $\begin{array}{l}\text { Virton, 2.IX.1920, M. } \\
\text { Goetghebuer }\end{array}$ \\
\hline & $\begin{array}{l}\text { Chironomus } \\
\text { (Cryptochironomus) } \\
\text { nigrofascia }\end{array}$ & FREEMAN, 1961 & $\begin{array}{l}\text { Holotype, male, } \\
\text { good }\end{array}$ & $\begin{array}{l}\text { Parachironomus } \\
\text { nigrofasciatus FREEMAN, } \\
1961\end{array}$ & $\begin{array}{l}\text { Madagascar Centre, } \\
\text { Pic Boby } 2400 \mathrm{~m}, \\
\text { Andringitra, } \\
\text { Ambalavao, 11- } \\
\text { 14.I.1958, B. } \\
\text { Stuckenberg }\end{array}$ \\
\hline & $\begin{array}{l}\text { Chironomus } \\
\text { (Endochironomus) } \\
\text { pruinosa }\end{array}$ & FREEMAN, 1961 & $\begin{array}{l}\text { Holotype, male, } \\
\text { good }\end{array}$ & \begin{tabular}{|l} 
Endochironomus \\
pruinosus FREEMAN ,1961
\end{tabular} & $\begin{array}{l}\text { Madagascar Nord, } \\
\text { Montagne Ambre } \\
\text { 1000m, det. Diego } \\
\text { Suarez, 23.XI- } \\
\text { 4.XII.1958, B. } \\
\text { Stuckenberg }\end{array}$ \\
\hline & Chironomus latidens & $\begin{array}{l}\text { GOETGHEBUER, } \\
1921\end{array}$ & $\begin{array}{l}\text { Cotype, male, } \\
\text { good }\end{array}$ & $\begin{array}{l}\text { Einfeldia pagana MEIGEN, } \\
1838\end{array}$ & $\begin{array}{l}\text { Destelbergen, } \\
\text { 1.VI.1920, M. } \\
\text { Goetghebuer }\end{array}$ \\
\hline & Chironomus latidens & $\begin{array}{l}\text { GOETGHEBUER, } \\
1921\end{array}$ & $\begin{array}{l}5 \text { specimens as } \\
\text { cotype but } \\
\text { without cotype } \\
\text { label }\end{array}$ & & \\
\hline
\end{tabular}




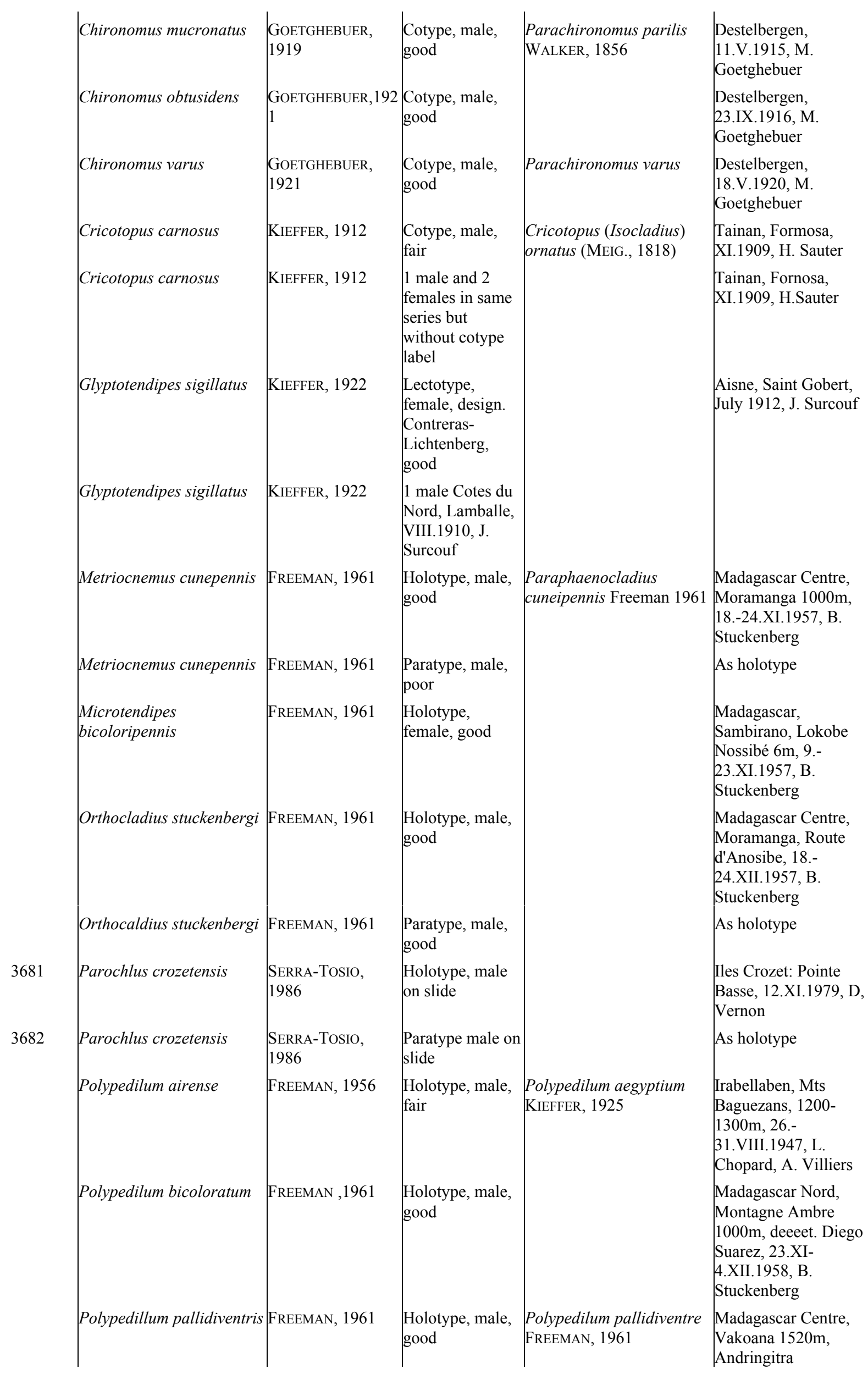




\begin{tabular}{|c|c|c|c|c|c|}
\hline & & & & & $\begin{array}{l}\text { Ambalavao, 21.- } \\
\text { 24.I.1958, B. } \\
\text { Stuckenberg }\end{array}$ \\
\hline & Polypedilum pelostolum & KIEFFER, 1912 & $\begin{array}{l}\text { Cotype, female, } \\
\text { fair }\end{array}$ & $\begin{array}{l}\text { Polypedilum nubifer } \\
\text { SKUSE } 1889\end{array}$ & $\begin{array}{l}\text { Tainan, Formosa, } \\
\text { X.1908, H. Sauter }\end{array}$ \\
\hline & Polypedilum pelostolum & KIEFFER, 1912 & $\begin{array}{l}2 \text { males and } 2 \\
\text { females in same } \\
\text { series but } \\
\text { without cotype } \\
\text { label }\end{array}$ & & \\
\hline & Polypedilum stuckenbergi & FREEMAN, 1961 & $\begin{array}{l}\text { Holotype, male, } \\
\text { good }\end{array}$ & & $\begin{array}{l}\text { Madagascar Centre, } \\
\text { Pic Boby 2400m, } \\
\text { Andringitra, } \\
\text { Ambalavao, 11- } \\
\text { 14.I.1958, B. } \\
\text { Stuckenberg }\end{array}$ \\
\hline & Polypedilum stuckenbergi & FREEMAN, 1961 & $\begin{array}{l}\text { Paratype, male, } \\
\text { good }\end{array}$ & & As holotype \\
\hline & Procladius formosanus & KIEFFER, 1912 & $\begin{array}{l}\text { Cotype, male, } \\
\text { fair }\end{array}$ & Tanypus formosanus & $\begin{array}{l}\text { Tainan, Formosa, } \\
\text { II.1909, H. Sauter }\end{array}$ \\
\hline 3741 & Smittia celtica & $\begin{array}{l}\text { ROSSARO \& } \\
\text { DELETTRE, } 1992\end{array}$ & $\begin{array}{l}\text { Paratype male on } \\
\text { slide }\end{array}$ & & $\begin{array}{l}\text { Paimpont, Brittany, } \\
\text { NL 13.XII.1988 }\end{array}$ \\
\hline 3740 & Smittia celtica & $\begin{array}{l}\text { ROSSARO \& } \\
\text { DELETTRE, } 1992\end{array}$ & $\begin{array}{l}\text { Paratype male on } \\
\text { slide }\end{array}$ & & $\begin{array}{l}\text { Paimpont, Brittany, } \\
\text { PA4 13.XII.1988 }\end{array}$ \\
\hline 3742 & Smittia celtica & $\begin{array}{l}\text { ROSSARO \& } \\
\text { DELETTRE, } 1992\end{array}$ & $\begin{array}{l}\text { Paratype larva on } \\
\text { slide }\end{array}$ & & $\begin{array}{l}\text { Paimpont, Brittany, } \\
\text { NPA 13.XII.1988 }\end{array}$ \\
\hline 3739 & Smittia celtica & $\begin{array}{l}\text { ROSSARO \& } \\
\text { DELETTRE, } 1992\end{array}$ & $\begin{array}{l}\text { Holotype male } \\
\text { on slide }\end{array}$ & & $\begin{array}{l}\text { Paimpont, Brittany, } \\
\text { NL 13.XII.1988 }\end{array}$ \\
\hline & Stenochironomus unicalar & FREEMAN, 1961 & $\begin{array}{l}\text { Holotype, } \\
\text { female, good }\end{array}$ & & $\begin{array}{l}\text { Madagascar Nord, } \\
\text { Montagne Ambre } \\
\text { 1000m, det. Diego } \\
\text { Suarez, 23.XI- } \\
\text { 4.XII.1958, B. } \\
\text { Stuckenberg }\end{array}$ \\
\hline & Tanytarsus formosanus & KIEFFER, 1912 & $\begin{array}{l}\text { Cotype, female, } \\
\text { fair }\end{array}$ & & $\begin{array}{l}\text { Tainan, Formosa, } \\
\text { II.1909, H. Sauter }\end{array}$ \\
\hline & Tanytarsus formosanus & KIEFFER, 1912 & $\begin{array}{l}2 \text { males and } 2 \\
\text { females in same } \\
\text { series but } \\
\text { without cotype } \\
\text { label }\end{array}$ & & $\begin{array}{l}\text { Tainan, Formosa, } \\
\text { II.1909 and X.1908, H. } \\
\text { Sauter }\end{array}$ \\
\hline & Tendipes tainanus & KIEFFER, 1912 & $\begin{array}{l}\text { Cotype, male, } \\
\text { good }\end{array}$ & Nilodorum tainanus & $\begin{array}{l}\text { Tainan, Formosa, } \\
\text { II.1909, H. Sauter }\end{array}$ \\
\hline & Tendipes tainanus & KIEFFER, 1912 & $\begin{array}{l}1 \text { male and } 2 \\
\text { females in same } \\
\text { series but } \\
\text { without cotype } \\
\text { label }\end{array}$ & & $\begin{array}{l}\text { Tainan, Formosa, } \\
\text { XI.1909 and II.1909, } \\
\text { H. Sauter }\end{array}$ \\
\hline
\end{tabular}

Table 2. Chironomid types in the Macquart collection

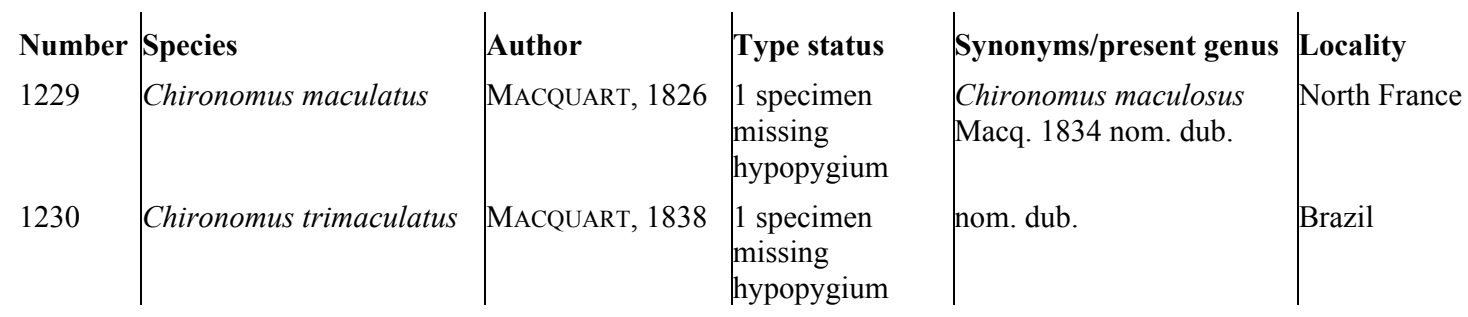




\begin{tabular}{l|l|l|l}
1231 & Chironomus ferrugineus & MACQUART, 1838 & 1 female \\
1232 & Chironomus maculipennis & BLANCHARD, 1852 & 3 males \\
1233 & Chironomus pallidulus & BLANCHARD, 1852 & 3 males \\
1234 & Chironomus obscurellus & BLANCHARD, 1852 & \\
1235 & $\begin{array}{l}\text { Chironomus tessellatus } \\
\text { missing } \\
\text { hypopygium }\end{array}$ \\
1236 & Chironomus articuliferus & BLANCHARD, 1852 & $\begin{array}{l}\text { BLANCHARD, } 1852 \\
1 \text { male } \\
3 \text { males }\end{array}$
\end{tabular}

\begin{tabular}{|l|l} 
nom. dub. & Brazil \\
Chironomus & Chile \\
maculosipennis Kieffer & \\
1906 replacement name. & \\
Unplaced in Chironiminae & \\
Jun. hom. of C. pallidulus & Chile \\
Meigen. Unplaced in & \\
Chironominae & \\
Unplaced in Chironominae & Chile \\
& \\
& \\
Unplaced in Chironominae & Chile \\
Unplaced in Chironomidae & Chile
\end{tabular}

